\title{
Research on Multipoint Leak Location of Gas Pipeline Based on Variational Mode Decomposition and Relative Entropy
}

\author{
Yongmei Hao ${ }^{1},{ }^{1}$ Zhanghao Du, ${ }^{1}$ Juncheng Jiang, ${ }^{1}$ ZhiXiang Xing, ${ }^{1}$ Xinming Yan, \\ Shuli Wang, ${ }^{1}$ and Yongchao Rao' \\ ${ }^{1}$ School of Environmental and Safety Engineering, Changzhou University, Changzhou 213164, China \\ ${ }^{2}$ Changzhou Branch of Jiangsu Special Equipment Safety Supervision and Inspection Institute, Changzhou 213161, China \\ Correspondence should be addressed to Yongmei Hao; hymzcs@cczu.edu.cn
}

Received 24 June 2020; Revised 22 November 2020; Accepted 3 December 2020; Published 31 December 2020

Academic Editor: Ehsan Ahmadi

Copyright (C) 2020 Yongmei Hao et al. This is an open access article distributed under the Creative Commons Attribution License, which permits unrestricted use, distribution, and reproduction in any medium, provided the original work is properly cited.

Pipeline leak detection has always been a relatively difficult technical problem; especially in urban pipeline leak detection, there are still many problems to be solved. A multipoint leak detection and location method for urban gas pipelines based on variational mode decomposition and relative entropy was proposed. Firstly, the experiment pipeline system was built, and the original signal was collected by acoustic emission technology; then, a variational model method was used to decompose the signal to obtain multiple intrinsic mode function (IMF) components with different characteristic scales. According to the characteristics of relative entropy, each component was analyzed, the appropriate IMF component was selected, and the selected component was reconstructed to obtain the observation signal. The multipoint leakage location model of the urban gas pipeline was established. The number of source signals was estimated by singular value decomposition, and the leakage signals were separated; finally, the accurate location of leakage point was achieved by cross-correlation positioning. The results showed that the average relative error of the pipeline leak location results is $2.97 \%$, and the leak location accuracy is significantly improved, achieving the purpose of precise location.

\section{Introduction}

Urban buried gas pipeline network, as one of the indispensable infrastructure of the city, has developed rapidly in many cities in recent years, and the scale of urban underground pipeline is getting larger. With the increase in the number of urban gas pipelines, the growth of operating time, and the aging of the pipe network, the safety problem of underground pipeline leakage has become increasingly prominent, and accidents occur frequently. Once the gas pipeline leaks, it is easy to cause fire, explosion, poisoning, environmental pollution, and other malignant accidents. The early leakage of the underground gas pipeline in most cities in China is often difficult to be discovered and repaired. When the development becomes a major accident, huge loss of life and property has been caused. Therefore, the initial leakage of the pipeline is an important issue that should be concerned. In actual working conditions, there are often two or more leakage sources in a section of pipelines, that is, multisource leakage. The multisource leakage signals affect each other, coupled with the inherent characteristics of the leakage signals and environmental noise interference; the accurate location of multisource leakage has become an unsolvable problem in the pipeline leakage location [1-3].

The traditional pipeline leak detection technologies are manual inspection method, portable instrumentation method, in-pipe detector method, and so on. These methods not only can not be continuously detected but also have high investment costs and large defects. While conventional nondestructive testing such as ultrasonic testing crawlers, magnetic flux leakage testing crawlers, and other related technologies are relatively mature and have high testing accuracy, the testing process of these testing technologies is point-by-point scanning, and the tested equipment must be discontinued, and the testing efficiency is low; it is difficult to detect the city's complex pipe network system [4-7]. The 
acoustic emission pipeline leak detection system has the advantages of real-time continuous measurement analysis, no need for digging and dismantling the pipeline, and so on. It is especially suitable for small leak detection of urban pipelines with circular laying, multinodes, and short distances, and the detection efficiency is high. However, the acoustic emission signals collected by the acoustic emission technology contain a large amount of environmental noise and other sudden nonleakage signals. The signal has frequency dispersion and is susceptible to noise interference. It is difficult to extract effective leakage signals, especially for the presence of many pipelines. In the case of a leakage source, there is still a large error in the detection and positioning accuracy of acoustic emission. Therefore, it is necessary to explore a processing method for decomposing and extracting the effective leakage signal of the acoustic emission pipeline to achieve accurate positioning of the pipeline at multiple points [8-10].

In recent years, time-frequency analysis methods have been widely used in the field of signal processing, but they all have their own limitations [11]. Huang et al. [12] initially proposed empirical mode decomposition (EMD) as an adaptive time-frequency method to decompose a complex signal into a set of inherent mode functions (IMFs). However, due to the intermittent components in the signal, modal aliasing occurs in the EMD decomposition results. Smith [13] proposed the local mean decomposition (LMD) method, which effectively improved the modal aliasing problem of EMD, but like the EMD method, the LMD method still has the problem of end effects [14]. Therefore, it is necessary to find a signal processing method to effectively solve the problem of modal aliasing. VMD is a new nonstationary signal processing method proposed by Kedadouche et al. [15] in 2016. It uses a variational model to decompose the processed signal and searches for the optimal solution. It is a completely nonrecursive model. It uses the optimal component of the model and the corresponding center frequency to effectively solve the end effect problem in LMD $[16,17]$ and the modal aliasing phenomenon after EMD decomposition $[18,19]$. In terms of multipoint leak location of pipelines, Verde [20,21] first proposed the use of fault detection stagger generators and the analysis of leak locations and applied the analytical redundancy method to achieve the location of two leaks in the pipeline. A model method based on the internal flow and pressure of the pipeline is proposed to detect and locate the multipoint leakage source of the pipeline. By establishing a static relationship matching the physical leakage position, the existence conditions for the stagger generator are met, thereby reducing the number of two nonconcurrent leakage parameters.. The average error of the two-point leakage location is $14.67 \%$, but continuous leakage location cannot be achieved; on this basis, Delgado et al. [22] uses the extended Kalman filter detection principle to establish a model to realize the detection and location of pipeline multipoint leakage sources. However, when the number of leakage points is too large, this method cannot effectively locate the multipoint leakage. Lazhar et al. [23] uses the test pipeline in the laboratory to solve the two-point leakage problem of the viscoelastic pipeline through the transient fluid model method. The pipeline leakage was located, and the two-point leakage positioning error was $4.2 \%$. Negrete and Verde [24] introduced a sliding mode observer to reconstruct the multipoint leakage signal in the pipeline to achieve the location of the leakage source, but the key point is detection. Only the precise pressure at the leak point can realize the positioning of multiple leaks.

Generally speaking, although people have made some progress in detecting and locating multipoint leakage sources in pipelines, there are not many methods to truly realize convenient, effective, and accurate multipoint leakage detection in pipelines, especially in engineering applications. EMD, LMD, and other methods have certain advantages in signal processing, but there are also many problems such as modal aliasing, end effects, and so on. Therefore, this paper combines previous studies to propose a method based on variational mode decomposition (VMD) and relative entropy. The multipoint leakage location method of urban pipelines uses the acoustic emission system to collect multipoint leakage source signals without excavation of the gas pipeline and uses the VMD method to decompose the leakage signals to effectively eliminate the interference of nonleakage signals such as noise. On this basis, a multipoint leakage location model for pipelines is established, and the leakage location of the pipeline is accurately calculated through crosscorrelation positioning.

The rest of this article is organized as follows. Section 2 introduces the principle and simulation analysis of the VMD method. Section 3 introduces the pipeline multisource leak location model. The content of Section 4 is to verify the effectiveness of this method for pipeline multisource leakage location through field experiments. Section 5 outlines the main conclusions of this paper.

\section{Signal Extraction Based on VMD and Relative Entropy}

2.1. Variational Mode Decomposition. The variational mode decomposition (VMD) was proposed by Kedadouche et al. in 2016. Different from EMD, VMD uses the variational model to decompose the processed signal and search for the optimal solution. It is a completely nonrecursion model. It can solve the mode mixing problem in EMD by using the optimal amplitude modulation-frequency modulation component and the corresponding center frequency required by the model, and it has a high precision decomposition function. Therefore, the number of decomposed components can be determined by the actual signal setting, and the effective IMF components can be obtained.

The VMD algorithm [25] can decompose complex actual signals into components of different scales, which can be expressed as follows:

$$
u_{k}(t)=A_{k}(t) \cos \left[\phi_{k}(t)\right]
$$

where $k$ is the number of components, $u_{k}$ is the modal function, $\phi_{k}(t)$ is the number of phases, where $\phi_{k}(t) \geq 0$, 
$A_{k}(t)$ is the instantaneous amplitude, and $\omega_{k}(t)$ is the instantaneous frequency; and the envelope $A_{k}(t) \geq 0$.

According to the Carson principle, the bandwidth estimation of IMF can be expressed as follows:

$$
B W_{A M-F M}=2\left(\Delta f+f_{A M}+f_{F M}\right),
$$

where $\Delta f$ is the maximum deviation of $\omega_{k}(t) ; f_{A M}$ is the offset rate for $\omega_{k}(t)$; and $f_{F M}$ is the highest frequency of $\omega_{k}(t)$.

Unlike the EMD method which uses cyclic filtering to solve iterative operations, when VMD obtains IMF components, it seeks the optimal solution from the signal distribution to the constraint variational model to solve IMF [25]. In the decomposition process, the center frequency $\omega_{k}$ of each component and the corresponding bandwidth estimate $B W_{A M-F M}$ are iteratively updated continuously, and the adaptive decomposition of the signal band is realized according to the frequency characteristics of the actual signal. In short, VMD is to decompose the signal $f$ into $K$ bandwidth modal function $u_{k}(t)$. The specific decomposition steps of the algorithm are as follows [26]:

(1) Calculate the unilateral spectrum of $u_{k}(t)$ and perform Hilbert transform on it to obtain the analytical signal.

$$
\left[\delta(t)+\frac{j}{\pi t}\right] * u_{k}(t)
$$

(2) By adjusting and carrying the parameters, the center frequency $e^{-j \omega_{k} t}$ of each mode is added, and the modal function is transformed to the corresponding fundamental frequency.

$$
\left[\delta(t)+\frac{j}{\pi t}\right] * u_{k}(t) e^{-j \omega_{k} t}
$$

(3) Calculate the bandwidth of each modal signal by the 2-norm square $L^{2}$ gradient, and the objective function of the constrained variational problem can be expressed as

$$
\left\{\begin{array}{l}
\min _{\left\{u_{k}\right\},\left\{\omega_{k}\right\}}\left\{\sum_{K}\left\|\partial_{t}\left[\left(\delta(t)+\frac{j}{\pi t}\right) * u_{k}(t)\right] \exp \left(-j \omega_{k} t\right)\right\|^{2}\right\}, \\
\text { s.t. } \quad \sum_{K} u_{k}=\mathrm{X}(t),
\end{array}\right.
$$

where $\partial_{t}$ is the partial derivative of the function with respect to time $t,\left\{u_{k}\right\}$ represents the K IMF components obtained by decomposition, and $\left\{\omega_{k}\right\}$ represents the center frequency of each component. $\partial(t)$ is the Dirichlet function, $j$ is the symbol of the imaginary number, and s.t. is the English abbreviation for constraint conditions.

(4) In order to obtain the optimal model, the constrained variational model is transformed into the unconstrained variational model, and the second penalty term $\alpha$ and the Lagrange operator $\lambda(t)$ are introduced. Here, $\alpha$ can guarantee the reconstruction accuracy of the signal, which generally takes the value of 2000 , and $\lambda(t)$ can guarantee the rigor of the constraint model. Therefore, the Lagrange formulation is as follows:

$$
L\left(\left\{u_{k}\right\},\left\{\omega_{k}\right\}, \lambda\right):=\alpha \sum_{K}\left\|\partial_{t}\left[\left(\delta(t)+\frac{j}{\pi t}\right) * u_{k}(t)\right] \exp \left(-j \omega_{k} t\right)\right\|^{2}+\left\|f(t)-\sum_{K} u_{k}(t)\right\|_{2}^{2}+\left\langle\lambda(t), f(t)-\sum_{K} u_{k}(t)\right\rangle .
$$

Find the effective change points of the Lagrange formulation $(L)$ by alternately updating $u_{k}^{n+1}, \omega_{k}^{n+1}$, and $\lambda^{n+1}$. On the whole, during the iterative solution of the variational model, the frequency center and bandwidth of each component are continuously updated until the iteration termination condition is met. In this iterative process, the VMD method can obtain $K$ components according to the frequency domain characteristics of the actual signal, effectively avoiding the mode mixing.

2.2. Relative Entropy Theory. The initial use of entropy originated from the description in the lower thermodynamics. The "entropy" was the meaning of change. It was later cited to describe the mixing degree of information and defined in mathematics as a general function of the probability distribution. In information theory, people use entropy to describe the degree of chaos in an information system and define it as the more chaotic and unstable a system is, the greater the entropy of the system will be. On the contrary, the less chaotic and stable the system is, the smaller the information content is and the smaller the entropy value of the system will be. In general, entropy is the one that describes the uncertainty of a random variable. Entropy is introduced into the signal characteristics of nonstationary randomness in signal information processing. It can express the characteristics and quantitative representation of signals at different levels. To this end, it is introduced into the characteristic description of the variational mode decomposition signal component, and then the useful signal component is extracted [26].

Relative entropy, also known as Kullback-Leibler divergence, or information gain, is originally proposed by 
Solomon Kullback and Richard Leibler in 1951. In probability theory and information theory, relative entropy is used to calculate the symmetry or difference of two distributions, which can describe the difference between two random probability distributions. When the relative entropy value is larger, it indicates that the difference of these two random probability distributions is greater and vice versa [27].

$$
S_{K L}(P \| Q)=\sum_{i} \ln \left(\frac{P(i)}{Q(i)}\right) P(i) .
$$

Among them, $P$ and $Q$, respectively, represent the probability distribution of the two signals and $S_{K L}$ represents the relative entropy.

\section{Pipeline Multisource Leak Location Model}

The single point leakage can directly realize the accurate location of the pipeline leakage by using the mutual timefrequency analysis method and the modal acoustic emission technology through the extraction of the effective leakage signal. However, when there are multiple leak points in the pipeline, the leakage location cannot be achieved on the extraction of the effective leakage signal, and the collected multisource leakage signal needs to be separated and extracted. Therefore, the single point leak location model detection method is not suitable for pipeline multisource leak detection and location.

Pipeline multisource leakage is not only interfered by nonleakage signals, noise signals, and sudden signals but also affected by signals between various leakage signals, which will lead to a large positioning error of multisource leakage. In addition, environmental noise and other factors influence the precise positioning of multisource leakage source. Therefore, a method is proposed to effectively solve the problem that the multisource leakage source signals of pipelines are affected by noise and nonleakage signals [28].

When two acoustic emission sensors installed on the pipeline are used to collect the acoustic emission signals of pipeline leakage and if there are multiple leaks, the signals received by the two sensors are the overlapping results of multiple leakage sources. Therefore, the independent component analysis technique is used to separate the mixed signal. In the theory of blind source separation, according to the relationship between the signal source and the output of the detection system, there is [29]

$$
\left\{\begin{array}{l}
x_{1}(n)=s_{1}(n)+s_{2}(n)+, \cdots,+s_{n}(n)+\eta_{1}(n), \\
x_{2}(n)=\varsigma_{1} s_{1}\left(n-t_{1}\right)+\varsigma_{2} s_{2}\left(n-t_{2}\right)+, \cdots,+\varsigma_{n} s_{n}\left(n-t_{n}\right)+\eta_{2}(n),
\end{array}\right.
$$

where $x_{1}(n)$ and $x_{2}(n)$ are signals received by sensor no. 1 and acoustic emission sensor no. 2 , respectively; $s_{1}(n)$ and $\varsigma_{1} s_{1}\left(n-t_{1}\right)$ are signals received by the acoustic emission sensor no. 1 and no. 2 , respectively, from the initial location signal of leakage source no. $1 ; s_{2}(n)$ and $s_{2} s_{2}\left(n-t_{2}\right)$ are signals received by the acoustic emission sensor no. 1 and no.
2 , respectively, from the initial location signal of leakage source no. $2 ; s_{n}(n)$ and $s_{n} s_{n}\left(n-t_{n}\right)$ are signals received by the acoustic emission sensor no. 1 and no. 2 from the initial location signal of leakage source no. $n$, respectively; $\varsigma_{1}, \varsigma_{2}$, and $\varsigma_{n}$ are attenuation constants; $s_{1}\left(n-t_{1}\right)$ and $s_{n}(n)$ are signals of no. 1 leakage source, no. 2 leakage source, and no. $n$ leakage source received by acoustic emission sensor, respectively; $\eta_{1}(n)$ and $\eta_{2}(n)$ are noise signals received by the two acoustic emission sensors; and $t_{1}, t_{2}$, and $t_{n}$ are the delay of the leakage signal. In the theory of blind source separation, in order to solve the problem of source signal separation, it is assumed that each source signal is independent of each other, and the independent signal is recovered from the mixed signal by using ICA technology to estimate the source signal.

Establishment of the multipoint leakage location model is as follows. In order to separate the signals in different positions from the multisource leakage signals, it is necessary to process the leakage signals after noise reduction. Before that, the number of source signals should be estimated to estimate the accurate leakage source signals. By using the effective leakage signal extraction method, the best observation signal $x_{d}(t)$ of multisource leakage is obtained. The singular value decomposition and eigenvalues are combined to estimate the number of source signals. In the eigenvalue calculation of singular value decomposition, when the estimated covariance matrix and the relative noise power are set to be small, the space dimension can be estimated by judging the foot mutation point; that is, the number of numerical values of the minimum eigenvalue can be used to determine the number of sources in the blind source signal $[28,30]$. According to the principle of pipeline leakage location, the leakage source signals at the same position have obvious correlation. The correlation between the two signals is strong, and the corresponding peak value is large. The correlation between the two signals is weak, and the corresponding peak value is small or even none. Therefore, it is necessary to conduct cross-correlation analysis on the separated pipeline leakage signal to determine whether the signal is from the same leakage source.

The specific steps of the pipeline multisource leak location model are as follows:

(1) Use the VMD-relative entropy adaptive effective leakage signal extraction method to obtain the optimal upstream observation signal $x_{d}(t)$ of the pipeline multisource leakage;

(2) Calculate the observed signal $x_{d}(t)$ correlation matrix and then perform singular value decomposition to obtain the eigenvalues and obtain the estimated number of sources;

(3) Centralize the optimal observation signal $x_{d}(t)$; obtain the estimated signal $Y_{91}(t)$ of the leak signal source of the upstream sensor no. 1; 
(4) Similarly, obtain the estimated signal $y$ of the downstream leakage signal source of the pipeline and calculate the correlation coefficient of $Y_{91}(t)$ and $Y_{92}(t)$

(5) Determine whether the leakage signal is the same leakage source signal and match it one by one. Use the time-frequency analysis relationship between the delay and frequency of the time-varying cross-correlation function to determine the delay and wave speed;

(6) The location of the pipeline leakage point can be determined by the time difference positioning method.

\section{Experimental Verification}

4.1. Introduction to the Experiment. The positioning test platform of pipeline leakage acoustic emission detection is mainly composed of a pipe network system and an acoustic emission detection system. The test pipeline consists of three U-shaped seamless steel pipes of different diameters. The specifications of the pipes are DN150, DN100, and DN65, and the total length is 135 meters. There is a section of a buried polyethylene pipe (PE pipe), which is connected to the downstream of the DN150 seamless steel pipe and is about 32 meters long. Control valves, liquid electromagnetic flowmeters, and gas vortex flowmeters are provided at the start and end points of the pipeline. At the end of the pipeline, there is a surge tank of 1 cubic meter. Pressure gauges, thermometers, and flowmeters are installed at the inlet and outlet of the pipeline.

As shown in the pipeline test site diagram of Figure 1, the seamless steel pipe test subject of DN150 is selected, and the size is $\varphi 165 \times 4.5 \mathrm{~mm}$. The gas tank is inflated by an air compressor, and the gas tank supplies a medium-low pressure airflow to the steel pipe. Among them, the pipeline pressure is $0.25 \mathrm{MPa}$. In order to simulate a more real leak, the leak hole is drilled directly in the pipeline, the leak hole diameter is $1.5 \mathrm{~mm}$, and the leak hole is shown in Figure 2(a). The three leakage holes are located $6 \mathrm{~m}, 16 \mathrm{~m}$, and $30 \mathrm{~m}$ upstream from the no. 1 sensor. As shown in Figure 2(b), the distance between the upstream and downstream $\mathrm{AE}$ sensors is $42 \mathrm{~m}$, and the type of the acoustic emission sensor is DP3I, and the amplifier gain is $40 \mathrm{~dB}$. The AE sensor is coupled to the outer wall of the pipe. In the field inspection of the urban underground gas pipeline, the sensor can be arranged at the end of the pipe section in the inspection well between the pipe sections, as shown in Figure 2(c). The sampling frequency of the acoustic emission instrument is set to $1 \mathrm{Msps}$, and the sampling length is $2 \mathrm{~K}$, that is, 2048 sampling points. After many times of trial testing, the threshold value is set to $35 \mathrm{~dB}$, to filter out part of the environmental noise. The multisource leakage location arrangement of the test pipeline is shown in Figure 3. During the test, the three leakage holes are opened simultaneously to collect the leakage acoustic emission signal.

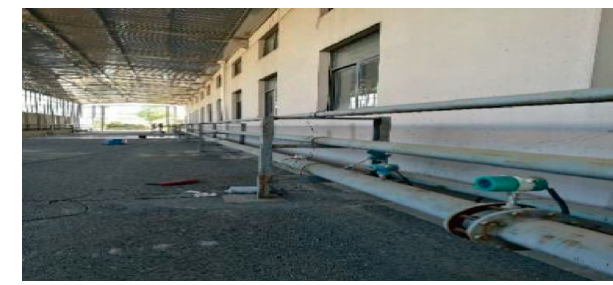

Figure 1: Pipeline multisource leakage test pipeline.

4.2. Data Processing and Signal Feature Extraction. In the pipeline leakage state, the original acoustic emission leakage signal is collected, and then the original leakage signal is analyzed through the acoustic emission detector; the results are shown in Figures 4(a)-4(f). Among them, Figures 4(a) and 4(b) are the waveform and spectrum of the initial location signal of pipeline multisource leakage detection, respectively, and Figures 4(c)-4(f) represent the signal RMS diagram, signal amplitude map, signal energy diagram, and signal ASL diagram of the initial leakage location. It can be seen from the diagram that when there is a multisource leakage in the local pipeline, the acoustic emission technology is difficult to locate effectively, and the error is large. Therefore, the signal is analyzed by Matlab programming. The time-frequency distribution of the upstream and downstream pipeline leakage original signal is obtained in Figure 5. Taking the original signal of the upstream no. 1 sensor as an example, the signal is divided into seven IMF components by VMD decomposition, each of which is shown in Figure 6.

Combined with the effective signal extraction method of relative entropy analysis, the observed signal is obtained, as shown in Figure 7. The source number estimation based on the singular value decomposition is performed, and the obtained eigenvalues are as shown in Figure 8. It can be seen from Figure 9 that the first three feature values are large. Therefore, the number of source signals is estimated to be three based on the correlation matrix eigenvalues.

As shown in Figure 9, the mixed signals are separated to obtain three estimated upstream and downstream leakage signals of the pipeline. The correlation coefficients of the separated original signal and the three separated signals are, respectively, calculated to be $0.8541,0.9064$, and 0.8503 ; the correlation coefficient between the estimated leakage signal downstream and the original signal is $0.8074,0.9464$, and 0.8792. It is known from the correlation coefficient that the effect of blind source separation is better. In the multisource leak location of the pipeline, the signals after the mixed signal separation are pipeline leakage signals. In order to distinguish whether the source of the signal is the same, the correlation between the three upstream no. 1 sensor signals and the three downstream signals is calculated by using the principle of cross correlation so as to distinguish whether the signals are from the same leakage source and obtain the corresponding upstream and downstream leakage signals. The results obtained are shown in Table 1.

It can be seen from Table 1 that the signal $Y_{\zeta_{1}}$ and the signal $Y_{\zeta 4}$ come from the same leakage source, the signal $Y_{\zeta 2}$ 


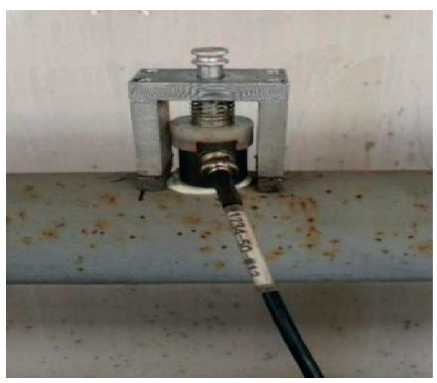

(a)

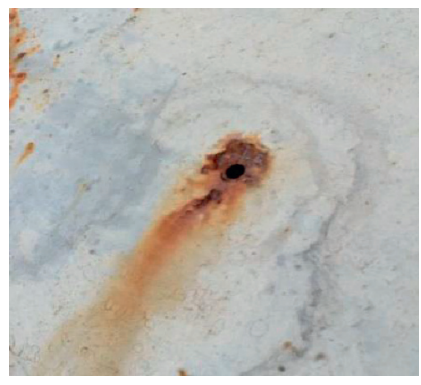

(b)

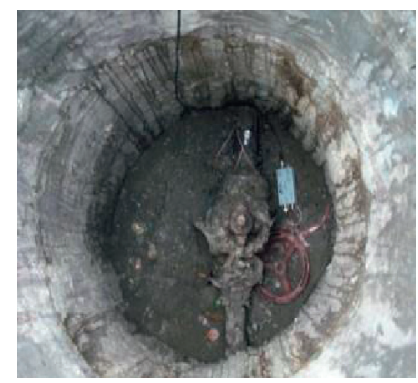

(c)

Figure 2: Acoustic emission detection system layout: (a) sensor arrangement, (b) leakage hole diagram, and (c) arrangement of field detection sensors.

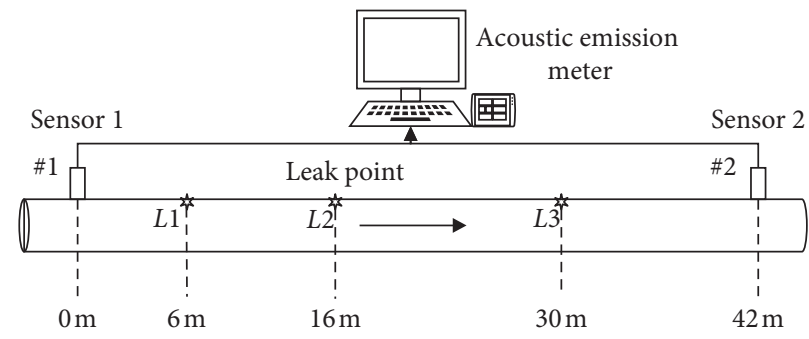

Figure 3: Test pipeline leakage location diagram.

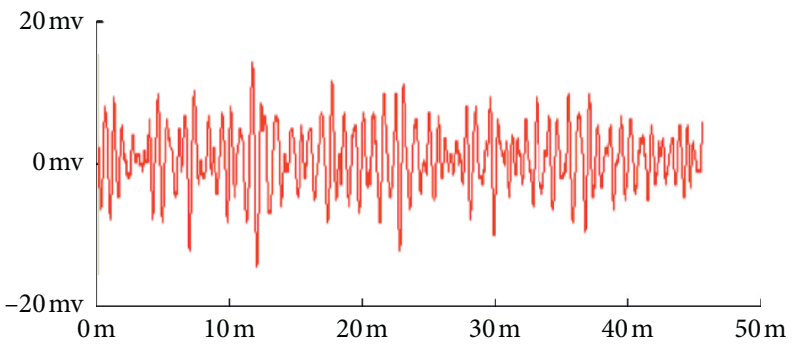

(a)

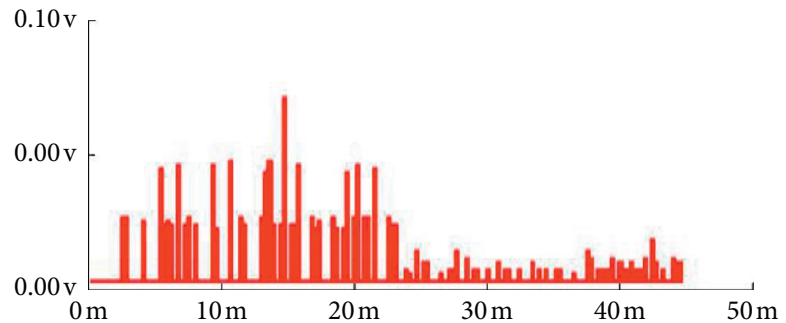

(c)

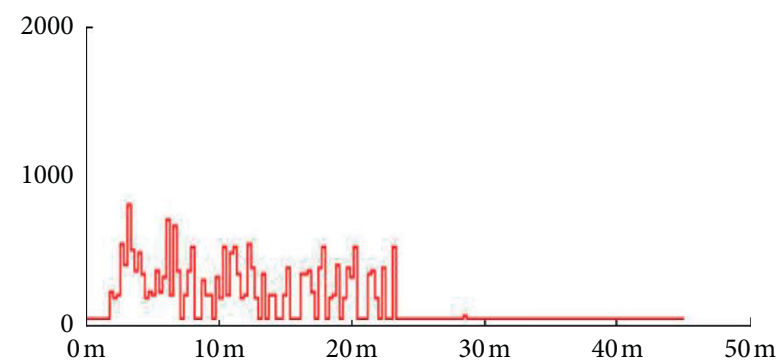

(e)

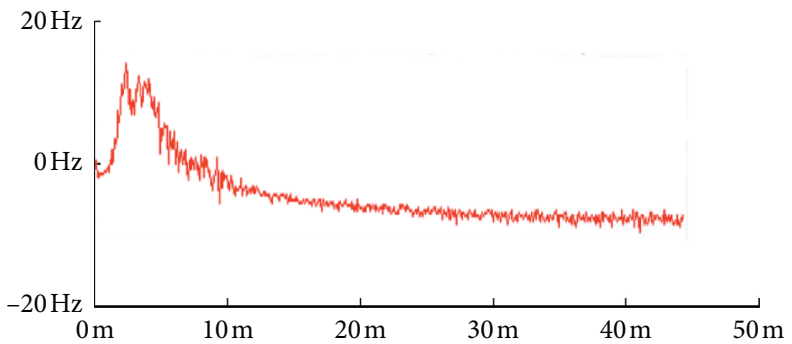

(b)

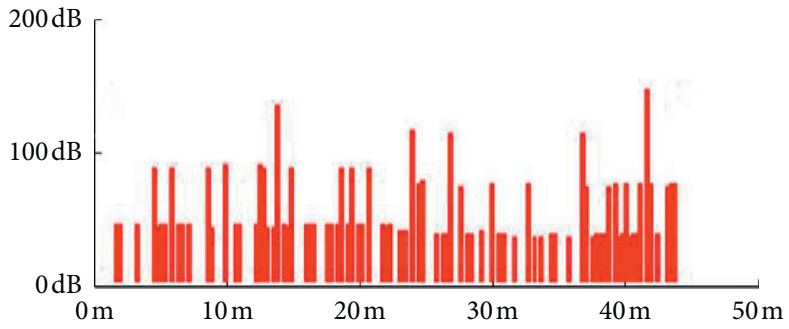

(d)

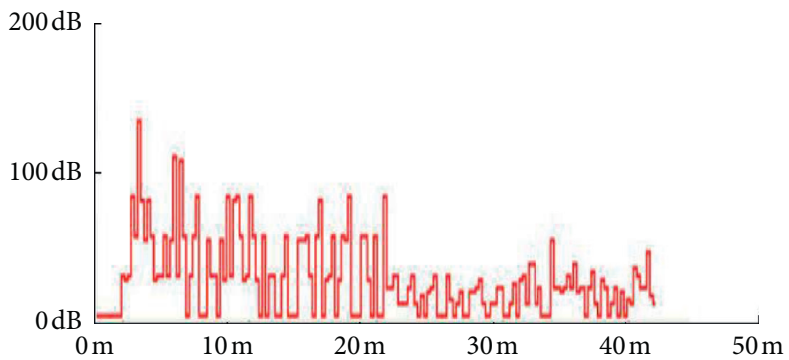

(f)

Figure 4: The result of using acoustic emission to deal with multisource leakage: (a) waveform of the initial positioning signal; (b) spectrum of the initial location signal; (c) RMS of the initial positioning signal; (d) amplitude of the initial positioning signal; (e) energy map of the initial positioning signal; (f) ASL of the initial positioning signal. 


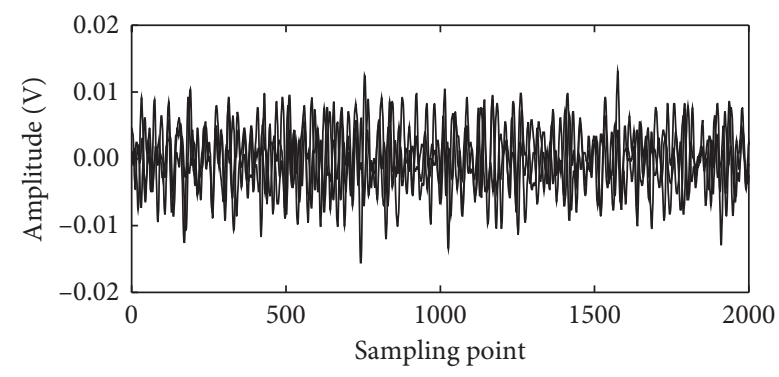

(a)

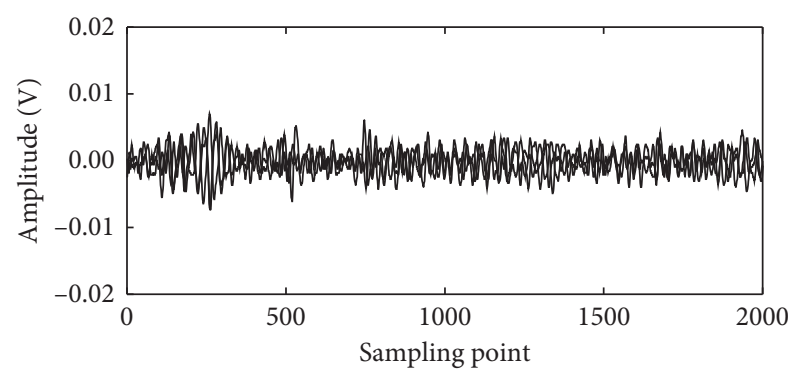

(b)

Figure 5: Pipeline leakage original signal: (a) upstream no. 1 sensor pipeline leakage original signal and (b) downstream no. 2 sensor pipeline leakage original signal.

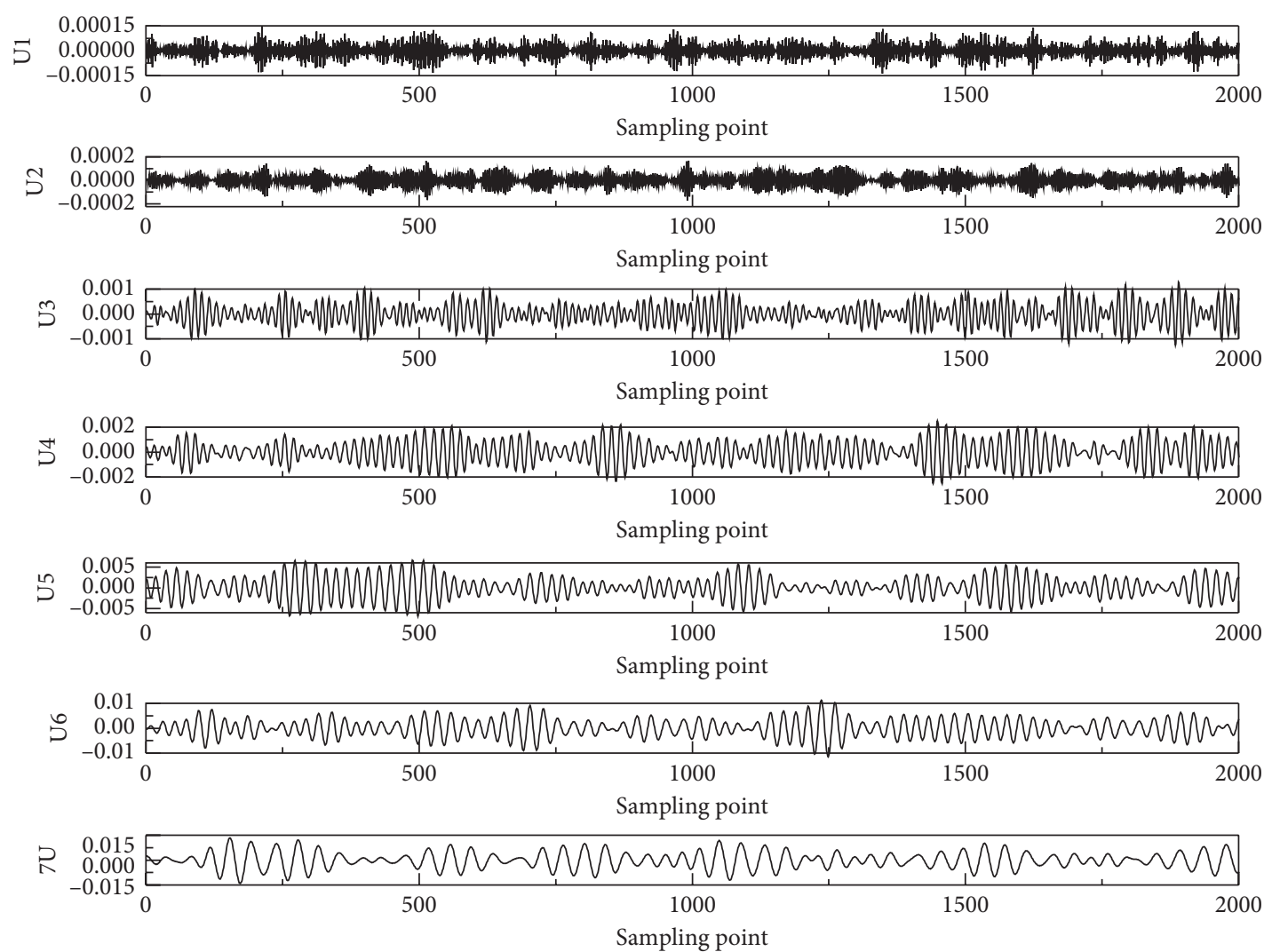

FIGURE 6: The components of VMD decomposes the upstream pipeline leakage original signal of the no. 1 sensor.

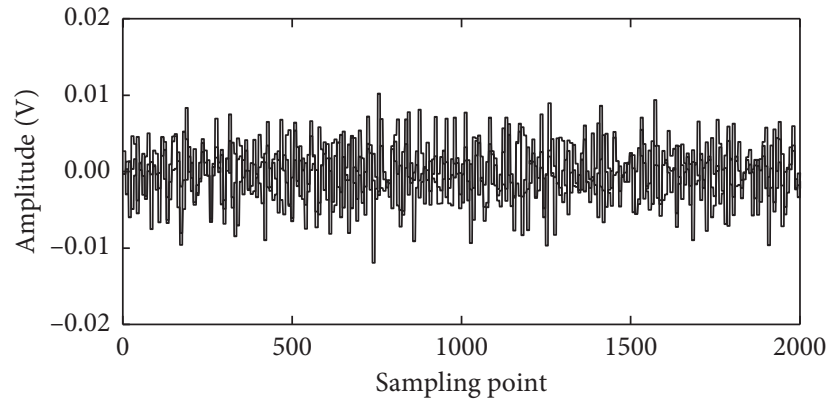

FIGURE 7: Upstream leakage noise reduction signal. 


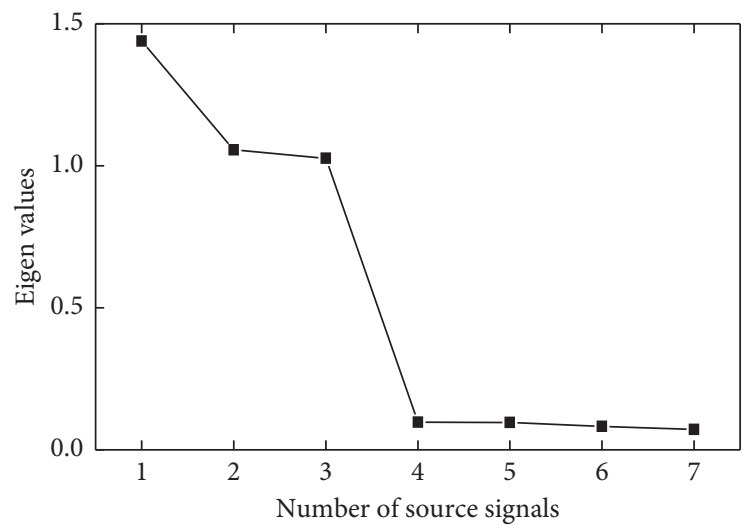

FIgURE 8: Correlation matrix eigenvalues.
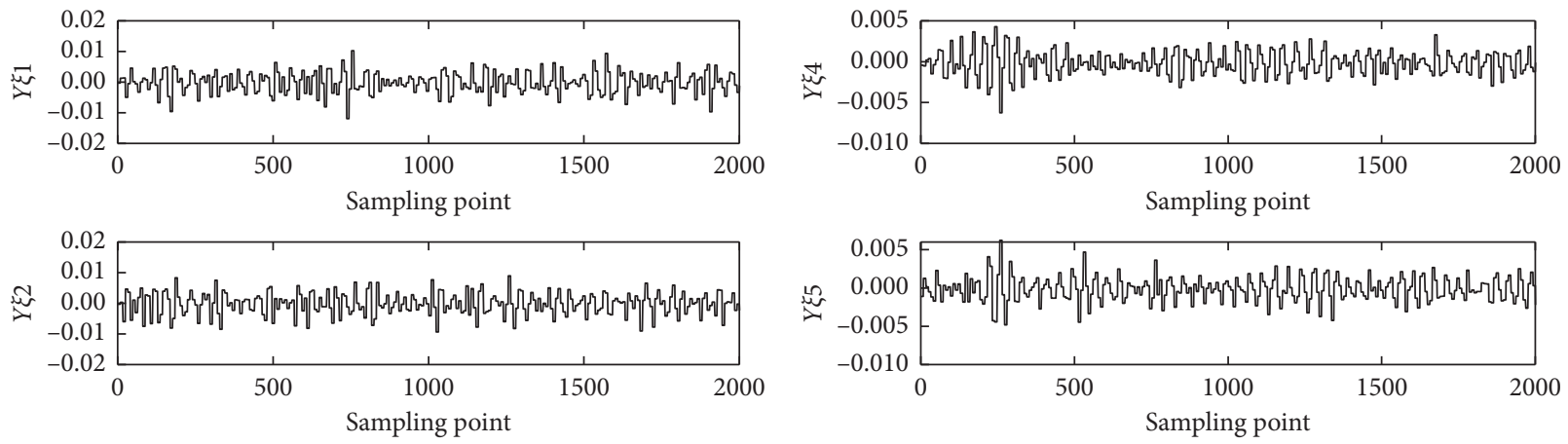

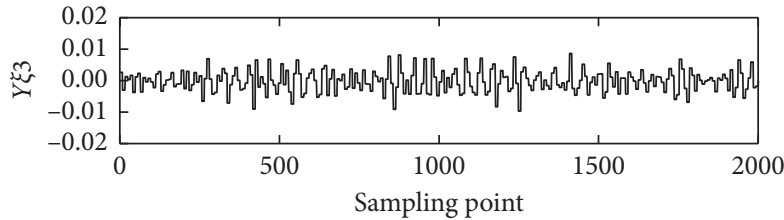

(a)

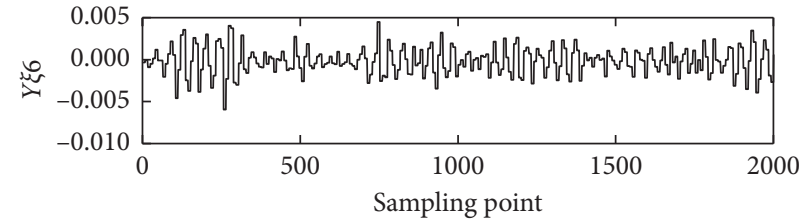

(b)

FIGURE 9: Estimated upstream and downstream leakage signals diagram: (a) upstream no. 1 sensor leakage noise reduction signal diagram and (b) downstream no. 2 sensor leakage noise reduction signal diagram.

TABLE 1: Two-two correlation coefficient between separated signals.

\begin{tabular}{lccc}
\hline Correlation coefficient & $Y_{\zeta 1}$ & $Y_{\zeta 2}$ & $Y_{\zeta 3}$ \\
\hline$Y_{\zeta 4}$ & 0.8341 & 0.3541 & 0.0254 \\
$Y_{\zeta 5}$ & 0.1398 & 0.1548 & 0.8653 \\
$Y_{\zeta 6}$ & 0.0987 & 0.8246 & 0.0398 \\
\hline
\end{tabular}

and the signal $Y_{\zeta 6}$ come from the same leakage source, and the signal $Y_{\zeta 3}$ and the signal $Y_{\zeta 5}$ come from the same leakage source so that the cross-correlation analysis can be performed on the corresponding signal.

4.3. Analysis of Results. In order to prove the effectiveness of this method, the direct mutual time-frequency analysis method, EMD-based component analysis method, and the method mentioned in this paper are, respectively, used to process the upstream and downstream leakage signals, and the delay and corresponding frequency of the pipeline leakage signal are calculated, and the results are shown in Figure 10.

It can be seen from Figure 10 that in the multisource leak pipeline detection, due to the interference of nonleakage signals such as noise, it is easy to generate multiple peaks and difficult to obtain the delay of the signal and the leak location by using the direct mutual time-frequency analysis method; based on the EMD analysis method, due to the mode mixing characteristics of EMD, the difference in signal identification is low so that the extracted signal also contains partial noise and produces errors; and the mode mixing is effectively solved by the method in the text. The characteristics eliminate the interference of nonleakage signals such as noise, extract the effective leakage signal to the maximum, and reduce the error of pipeline leakage location. For comparison, the multisource leak location experiment of multiple pipelines was carried out with the pipeline pressure of $0.1 \mathrm{MPa}, 0.15 \mathrm{MPa}$, and $0.25 \mathrm{MPa}$, respectively, and the detailed results of the calculation are shown in Table 2. 


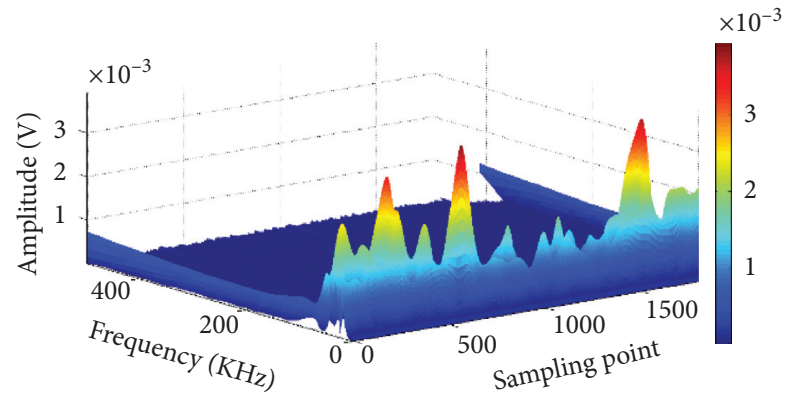

(a)

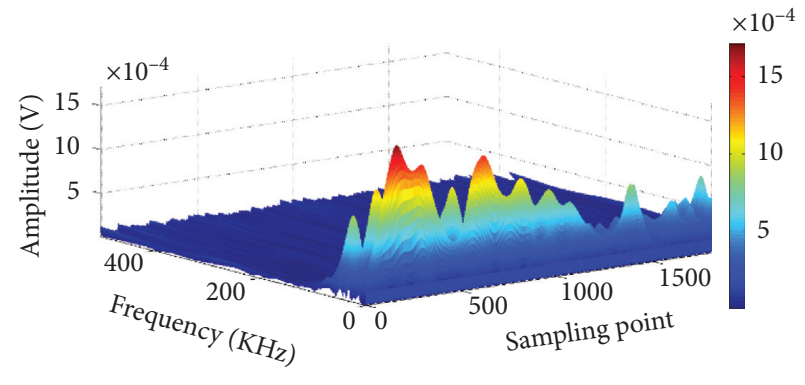

(b)

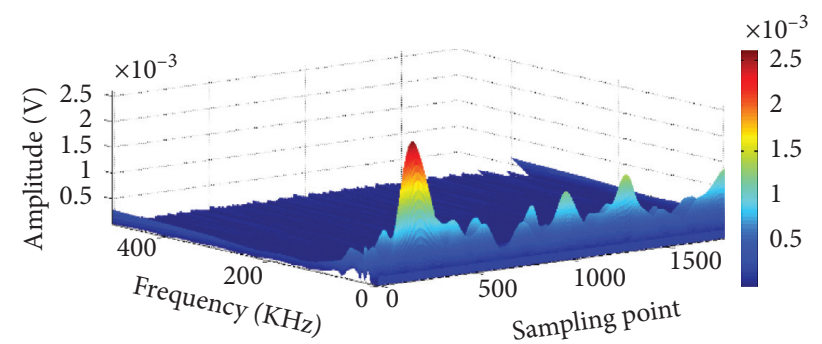

(c)

FIGURE 10:3D time-frequency diagram of different methods: (a) direct mutual time-frequency analysis method; (b) EMD method; (c) VMD method.

TABLE 2: Multisource leak location results under different pressures.

\begin{tabular}{|c|c|c|c|c|c|c|c|c|}
\hline \multirow[b]{2}{*}{ Number } & \multirow[b]{2}{*}{$\begin{array}{l}\text { Pressure } \\
(\mathrm{MPa})\end{array}$} & \multirow[b]{2}{*}{$\begin{array}{c}\text { True positioning } \\
\text { value }(\mathrm{m})\end{array}$} & \multicolumn{3}{|c|}{ The proposed method } & \multicolumn{3}{|c|}{ EMD method } \\
\hline & & & $\begin{array}{c}\text { Detection } \\
\text { location value } \\
(\mathrm{m})\end{array}$ & $\begin{array}{l}\text { Absolute } \\
\text { error }(m)\end{array}$ & $\begin{array}{l}\text { Relative } \\
\text { error (\%) }\end{array}$ & $\begin{array}{c}\text { Detection } \\
\text { location value } \\
(\mathrm{m})\end{array}$ & $\begin{array}{l}\text { Absolute } \\
\text { error }(\mathrm{m})\end{array}$ & $\begin{array}{l}\text { Relative } \\
\text { error (\%) }\end{array}$ \\
\hline 1 & 0.1 & 6 & 6.11 & 0.11 & 1.83 & 5.45 & 0.55 & 9.17 \\
\hline 2 & 0.1 & 6 & 6.14 & 0.14 & 2.33 & 5.55 & 0.45 & 7.50 \\
\hline 3 & 0.1 & 6 & 6.13 & 0.13 & 2.17 & 6.49 & 0.49 & 8.17 \\
\hline 4 & 0.15 & 6 & 5.48 & 0.52 & 8.67 & 6.39 & 0.39 & 6.50 \\
\hline 5 & 0.15 & 6 & 5.83 & 0.17 & 2.83 & 5.98 & 0.02 & 0.33 \\
\hline 6 & 0.15 & 6 & 5.74 & 0.26 & 4.33 & 5.37 & 0.63 & 10.50 \\
\hline 7 & 0.25 & 6 & 6.17 & 0.17 & 2.83 & 6.48 & 0.48 & 8.00 \\
\hline 8 & 0.25 & 6 & 6.42 & 0.42 & 7.00 & 5.13 & 0.87 & 14.50 \\
\hline 9 & 0.25 & 6 & 6.23 & 0.23 & 3.83 & 5.94 & 0.06 & 1.00 \\
\hline 10 & 0.25 & 6 & 6.44 & 0.44 & 7.33 & 6.56 & 0.56 & 9.33 \\
\hline 11 & 0.1 & 16 & 15.81 & 0.19 & 1.19 & 14.86 & 1.14 & 7.13 \\
\hline 12 & 0.1 & 16 & 15.09 & 0.91 & 5.69 & 14.68 & 1.32 & 8.25 \\
\hline 13 & 0.1 & 16 & 15.84 & 0.16 & 1.00 & 17.89 & 1.89 & 11.81 \\
\hline 14 & 0.15 & 16 & 16.28 & 0.28 & 1.75 & 16.98 & 0.98 & 6.13 \\
\hline 15 & 0.15 & 16 & 16.32 & 0.32 & 2.00 & 16.49 & 0.49 & 3.06 \\
\hline 16 & 0.15 & 16 & 16.29 & 0.29 & 1.81 & 18 & 2.00 & 12.50 \\
\hline 17 & 0.25 & 16 & 16.32 & 0.32 & 2.00 & 15.02 & 0.98 & 6.13 \\
\hline 18 & 0.25 & 16 & 15.83 & 0.17 & 1.06 & 15.69 & 0.31 & 1.94 \\
\hline 19 & 0.25 & 16 & 16.19 & 0.19 & 1.19 & 16.08 & 0.08 & 0.50 \\
\hline 20 & 0.25 & 16 & 16.48 & 0.48 & 3.00 & 16.99 & 0.99 & 6.19 \\
\hline 21 & 0.1 & 30 & 29.26 & 0.74 & 2.47 & 28.33 & 1.67 & 5.57 \\
\hline 22 & 0.1 & 30 & 29.20 & 0.80 & 2.67 & 27.34 & 2.66 & 8.87 \\
\hline 23 & 0.1 & 30 & 29.05 & 0.95 & 3.17 & 28.41 & 1.59 & 5.30 \\
\hline 24 & 0.15 & 30 & 30.32 & 0.32 & 1.07 & 27.39 & 2.61 & 8.70 \\
\hline 25 & 0.15 & 30 & 30.95 & 0.95 & 3.17 & 34.52 & 4.52 & 15.07 \\
\hline 26 & 0.15 & 30 & 31.13 & 1.13 & 3.77 & 31.89 & 1.89 & 6.30 \\
\hline 27 & 0.25 & 30 & 29.26 & 0.74 & 2.47 & 28.31 & 1.69 & 5.63 \\
\hline 28 & 0.25 & 30 & 30.51 & 0.51 & 1.70 & 25.98 & 4.02 & 13.40 \\
\hline 29 & 0.25 & 30 & 30.54 & 0.54 & 1.80 & 28.6 & 1.40 & 4.67 \\
\hline 30 & 0.25 & 30 & 29.13 & 0.87 & 2.90 & 27.36 & 2.64 & 8.80 \\
\hline
\end{tabular}




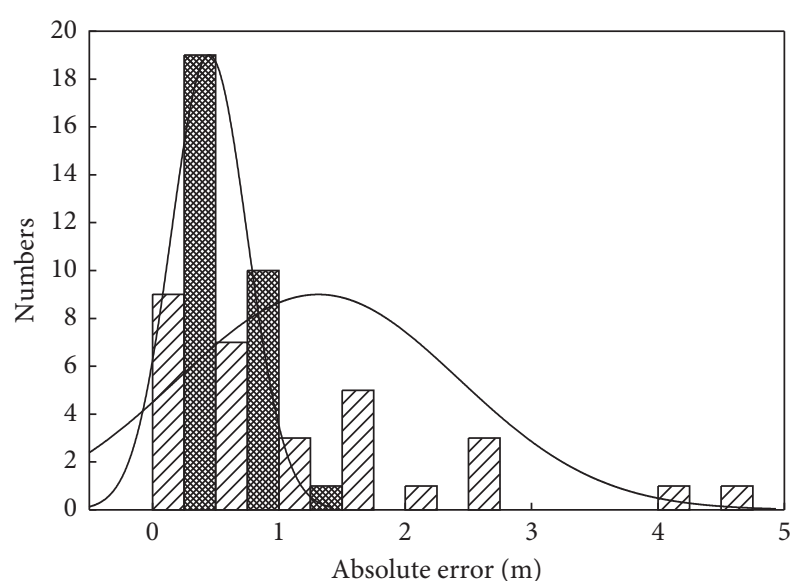

ZIIA EMD method

Proposed method

Figure 11: Pipe absolute positioning error.

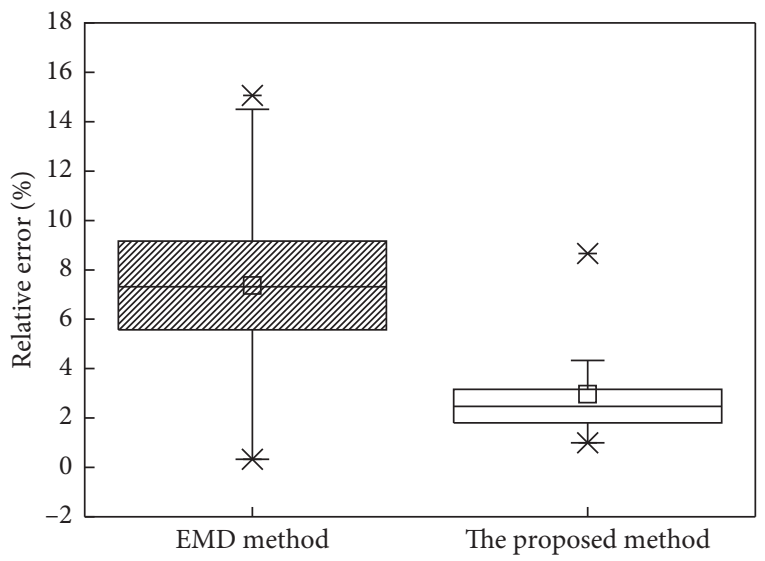

FIGURE 12: Relative positioning error distribution.

Among them, the absolute error is the difference between the detected positioning value and the real positioning value, and the unit is " $\mathrm{m}$ "; the relative error is the ratio of the absolute value of the absolute error to the real positioning value, and the unit is "\%."

Through the multiple sets of positioning results in Table 2, the absolute and relative errors of positioning are summarized, as shown in Figures 11 and 12. It can be seen from the diagram that the positioning error of the proposed method is normally distributed with an average absolute positioning error of $0.47 \mathrm{~m}$, and the distribution is dense and compact, and the relative locating error is $2.97 \%$; the average absolute locating error of the EMD-based analysis method is a normal distribution centered on $1.31 \mathrm{~m}$. The distribution range is wide and not concentrated enough. The relative positioning error is $7.36 \%$. Therefore, according to the multisource leakage model, the relative locating error based on the VMD-relative entropy method proposed in this paper is less than 3.4\% [23], which is compared with the relative locating error of the multisource leakage location method proposed by Ayed Lazhar based on the model location method, and the locating accuracy is higher.

\section{Conclusion}

Aiming at the problem that the detection and location accuracy of multipoint leakage of urban gas pipelines is not high, this paper proposes a multipoint leakage location method for pipelines based on VMD and relative entropy.

(1) In terms of acoustic emission signal feature extraction, compared with traditional acoustic emission technology, this method eliminates the influence of noise and other unrelated signals in the signal acquisition process by VMD and extracts the characteristics of the signal according to the sample entropy value of each component.

(2) In terms of multipoint leakage localization of urban gas pipelines, establish a multipoint leakage localization model for pipelines and obtain each estimated signal by ICA blind source separation of multiple leak source signals and then analyze the estimated signals to identify each leak source, and finally the cross-correlation location calculation is performed on the leakage source.

(3) After experimental verification, the average relative error based on the signal feature extraction and multipoint leakage positioning method proposed in this paper is $2.97 \%$. Compared with the EMD method, the average relative positioning error of the method proposed in this paper is significantly reduced. Therefore, the method proposed in this paper can effectively identify and locate leaks at multiple points in urban gas pipelines.

\section{Data Availability}

The data used to support the findings of this study are available from the corresponding author upon request.

\section{Conflicts of Interest}

The authors declare that they have no conflicts of interest.

\section{Acknowledgments}

This research was supported by the National Natural Science Foundation of China (51834007), the Subproject of National Key R \& D Plan (2019YFC0810700), the Key R \& D Projects of Jiangsu Province (BE2018642), and the Changzhou Science and Technology Support Plan (CE20185024).

\section{References}

[1] P.-S. Murvay and I. Silea, "A survey on gas leak detection and localization techniques," Journal of Loss Prevention in the Process Industries, vol. 25, no. 6, pp. 966-973, 2012.

[2] J. Sun, Q. Xiao, J. Wen, and F. Wang, "Natural gas pipeline small leakage feature extraction and recognition based on 
LMD envelope spectrum entropy and SVM," Measurement, vol. 55, no. 9, pp. 434-443, 2014.

[3] J. Li, M. Li, and J. Zhang, "Rolling bearing fault diagnosis based on time-delayed feedback monostable stochastic resonance and adaptive minimum entropy deconvolution," Journal of Sound and Vibration, vol. 401, pp. 139-151, 2017.

[4] M.-S. Kim and S.-K. Lee, "Detection of leak acoustic signal in buried gas pipe based on the time-frequency analysis," Journal of Loss Prevention in the Process Industries, vol. 22, no. 6, pp. 990-994, 2009.

[5] M. Firla, Z.-Y. Li, N. Martin, C. Pachaud, and T. Barszcz, "Automatic characteristic frequency association and allsideband demodulation for the detection of a bearing fault," Mechanical Systems and Signal Processing, vol. 80, pp. 335348, 2016.

[6] A. Aljaroudi, F. Khan, A. Akinturk, M. Haddara, and P. Thodi, "Probability of detection and false detection for subsea leak detection systems: model and analysis," Journal of Failure Analysis and Prevention, vol. 15, no. 6, pp. 873-882, 2015.

[7] L. Ni, J. Jiang, Y. Pan, and Z. Wang, "Leak location of pipelines based on characteristic entropy," Journal of Loss Prevention in the Process Industries, vol. 30, no. 1, pp. 24-36, 2014.

[8] F. Wu and L. Qu, "Diagnosis of subharmonic faults of large rotating machinery based on EMD," Mechanical Systems and Signal Processing, vol. 23, no. 2, pp. 467-475, 2009.

[9] Z. Wu and N. E. Huang, "Ensemble empirical mode decomposition: a noise-assisted data analysis method," $A d$ vances in Adaptive Data Analysis, vol. 1, no. 1, pp. 31-41, 2009.

[10] A. Tabrizi, L. Garibaldi, A. Fasana, and S. Marchesiello, "Early damage detection of roller bearings using wavelet packet decomposition, ensemble empirical mode decomposition and support vector machine," Meccanica, vol. 50, no. 3, pp. 865-874, 2015.

[11] M. A. Colominas, G. Schlotthauer, and M. E. Torres, "Improved complete ensemble EMD: a suitable tool for biomedical signal processing," Biomedical Signal Processing and Control, vol. 14, no. 1, pp. 19-29, 2014.

[12] N. E. Huang, Z. Shen, S. R. Long et al., "The empirical mode decomposition and the Hilbert spectrum for nonlinear and non-stationary time series analysis," in Proceedings of the Royal Society of London. Series A: Mathematical, Physical and Engineering Sciences, vol. 454, no. 1971, pp. 903-995, 1998.

[13] J. S. Smith, "The local mean decomposition and its application to EEG perception data," Journal of the Royal Society Interface, vol. 2, no. 5, pp. 443-454, 2005.

[14] Y. Yang, J. Cheng, and K. Zhang, "An ensemble local means decomposition method and its application to local rub-impact fault diagnosis of the rotor systems," Measurement, vol. 45, no. 3, pp. 561-570, 2012.

[15] M. Kedadouche, M. Thomas, and A. Tahan, "A comparative study between empirical wavelet transforms and empirical mode decomposition methods: application to bearing defect diagnosis," Mechanical Systems and Signal Processing, vol. 81, no. 2, pp. 88-107, 2016.

[16] R. Isermann, "Process fault detection based on modeling and estimation methods-a survey," Automatica, vol. 20, no. 4, pp. 387-404, 1984.

[17] C. Verde, L. Torres, and O. González, "Decentralized scheme for leaks' location in a branched pipeline," Journal of Loss Prevention in the Process Industries, vol. 43, pp. 18-28, 2016.

[18] C.-W. Liu, Y.-X. Li, Y.-K. Yan, J.-T. Fu, and Y.-Q. Zhang, "A new leak location method based on leakage acoustic waves for oil and gas pipelines," Journal of Loss Prevention in the Process Industries, vol. 35, pp. 236-246, 2015.
[19] C. Chuanbo, J. Shuguang, and H. X. Kevin, "Experimental study on the location of gas drainage pipeline leak using cellular automata," Journal of Loss Prevention in the Process Industries, vol. 95, no. 4, pp. 17-23, 2018.

[20] C. Verde, "Multi-leak detection and isolation in fluid pipelines," Control Engineering Practice, vol. 9, no. 6, pp. 673-682, 2001.

[21] C. Verde, "Accommodation of multi-leak location in a pipeline," Control Engineering Practice, vol. 13, no. 8, pp. 1071-1078, 2005.

[22] J. A. Delgado, G. Besancon, O. Begovich, and J. E. Carvajala, "Multi-leak diagnosis in pipelines based on extended kalman filter," Control Engineering Practice, vol. 49, no. 10, pp. 139-148, 2016.

[23] A. Lazhar, L. Hadj-Taïeb, and E. Hadj-Taïeb, "Two leaks detection in viscoelastic pipeline systems by means of transient," Journal of Loss Prevention in the Process Industries, vol. 26, no. 6, pp. 1341-1351, 2013.

[24] M. A. Negrete and C. Verde, "Multi-leak reconstruction in pipelines by sliding mode observers*," IFAC Proceedings Volumes, vol. 45, no. 20, pp. 934-939, 2012.

[25] Z. Ma, X. Liu, and Y. Li, "Rolling bearing fault feature extraction based on variational mode decomposition-wavelet packet transform and energy operator demodulation," Journal of Graphics, vol. 38, no. 2, pp. 174-179, 2017.

[26] S. Kullback and J. C. Keegel, "The minimum discrimination information approach to the analysis of contingency tables," Computational Statistics \& Data Analysis, vol. 3, no. none, pp. 133-141, 1985.

[27] R. B. Randall and J. Antoni, "Rolling element bearing diagnostics-a tutoria," Mechanical Systems and Signal Processing, vol. 25, no. 2, pp. 485-520, 2011.

[28] C. Wang, J. Xu, and Y. Zeng, "Research on influence of source number estimation on application of blind source separation algorithms," Procedia Computer Science, vol. 107, pp. 379384, 2017.

[29] X.-F. Xu, C.-D. Duan, L.-J. Liu, and X.-J. Yang, “A multi-stage algorithm for blind source separation," Optik, vol. 127, no. 7, pp. 3655-3659, 2016.

[30] M. E. Rhabi, H. Fenniri, A. Keziou, and E. Moreau, "A Robust algorithm for convolutive blind source separation in presence of noise," Signal Processing, vol. 93, no. 4, pp. 818-827, 2013. 